\title{
Burn-Out Chez Le Personnel Soignant Des Unités De Soins Intensifs De L'hôpital Universitaire De Parakou Au Bénin
}

\author{
Tchaou Blaise Adelin, \\ Unité d'Enseignement, de Formation et de Recherche en Anesthésie- \\ Réanimation et en Médecine d'Urgence \\ Djidonou Anselme, \\ Unité d'Enseignement, de Formation et de Recherche en Psychologie \\ Médicale et en Psychiatrie \\ Tchegnonsi N'venonfon Charles Frédéric, \\ Gbogblenou Gloria Todègnon Armistice, \\ Unité d'Enseignement, de Formation et de Recherche en Anesthésie- \\ Réanimation et en Médecine d'Urgence \\ Gandaho Prosper, \\ Unité d'Enseignement, de Formation et de Recherche en Psychologie \\ Médicale et en Psychiatrie
}

Doi: 10.19044/esj.2018.v14n24p408 URL:http://dx.doi.org/10.19044/esj.2018.v14n24p408

\begin{abstract}
The aim of the study was to identify Burn-out factors among caregivers in intensive care units in University Hospital of Parakou. We used a descriptive, analytical and correlational study with prospective data collection carried out over four months (March 1 to June 30, 2015) and 123 caregivers concerned consenting to the study. The Maslach Burn-out Inventory (MBI) was used for the assessment of burnout of the respondents. Among the 143 agents identified, 123 of them (86\%) consented to the survey. The average age of caregivers was $34.4 \pm 9.3$ years with a male predominance $(56.9 \%)$. Seventy-six caregivers lived with a partner $(61.8 \%)$ and $90.8 \%$ of them were monogamous. Seventy-one caregivers $(57.7 \%)$ were not satisfied with their socio-economic situations and $88.7 \%$ of them associate secondary activities. Eighty eight caregivers $(71.5 \%)$ were identified in their industry stressful conditions. The frequency of Burn-out was $68.3 \%$. The occurrence of burn out was associated with sex $(p=0.001)$, type of family $(p=0.001)$, vocational qualification $(\mathrm{p}=0.001)$ in the industry $(\mathrm{p}=0$ 001), socio-economic conditions $(\mathrm{p}=0.01)$, disease appeared in the exercise of the profession $(\mathrm{p}=$ $0.02)$, organizational factors $(\mathrm{p}=0.001)$ and stressful conditions $(\mathrm{p}=0.04)$. In
\end{abstract}


conclusion we observe that the function nurse in the intensive care unit of the University Hospital of Parakou has a physical and mental hardship among caregivers inducing a high score of Burn-out, implementing an adapted prevention program is therefore necessary to lowering it.

Keywords: Burn-out, Caregivers, Intensive Care Units, Stress Factors.

\section{Resume}

L'étude a pour objectif d'identifier les facteurs associés au Burn-out chez le personnel soignant des unités de soins intensifs de l'hôpital universitaire de Parakou. Elle est de type descriptif et analytique et la méthode est basée sur le recueil prospectif des données réalisé du 1er mars au 30 juin 2015 auprès de 123 soignants. Le Maslach Burn-out Inventory a servi à l'évaluation du syndrome d'épuisement professionnel des enquêtés. Les résultats obtenus font ressortir que sur 143 agents recensés, 123 (soit 86\%) avaient consenti à l'enquête. L'âge moyen des soignants était de 34,4 \pm 9,3 ans avec une prédominance masculine (56,9\%). Soixante-seize soignants vivaient en couple $(61,8 \%)$ et $90,8 \%$ d'entre eux étaient monogames. Soixante-onze soignants $(57,7 \%)$ n'étaient pas satisfaits de leurs situations socio-économiques et $88,7 \%$ d'entre eux associaient des activités secondaires. Quatre-vingt-huit soignants $(71,5 \%)$ avaient identifié dans leur secteur d'activité des pathologies stressantes. La fréquence du Burn-out était de $68,3 \%$. La survenue du Burn-out était associé au sexe $(\mathrm{p}=0,001)$, au type de famille $(\mathrm{p}=0,001)$, à la qualification professionnelle $(\mathrm{p}=0,001)$, au secteur d'activité ( $p=0,001)$, aux conditions socio-économiques $(p=0,01)$, aux maladies apparues en cours d'exercice du métier $(p=0,02)$, aux facteurs organisationnels $(p=0,001)$ et aux pathologies stressantes $(p=0,04)$. En conclusion, il ressort que la fonction soignante dans les unités de soins intensifs comporte une pénibilité physique et mentale induisant chez les soignants un score élevé du Burn-out qu'il importe d'abaisser par la mise en œuvre d'un programme adapté de prévention.

Mots clefs: Burn-out, Soignants, Unités de soins intensifs, Facteurs de Stress

\section{Introduction}

Le Burn-out est un syndrome d'épuisement professionnel caractérisé par une fatigue physique et psychique intense, généré par des sentiments d'impuissance et de désespoir. Le personnel des unités de soins intensifs, confronté aux souffrances des patients admis aux urgences ou au décès de certains d'entre eux est plus à même de développer ce syndrome. Ces soignants sont exposés à des évènements traumatisants qui tout en augmentant leur niveau de stress, peuvent être la cause des problèmes psychologiques et 
émotionnels ; lesquels peuvent conduire à l'épuisement professionnel (Kirouac, 2012). Selon le Bureau International du Travail (BIT), le stress est devenu l'un des plus graves problèmes de notre temps. Plusieurs pathologies à savoir les maladies cardio-vasculaires, l'ulcère gastrique et duodénal, la dépression peuvent trouver leur origine dans l'excès de stress en milieu professionnel (Bureau International du Travail, 1993). En France (Embriaco et al., 2007, et Poncet et al., 2007) ont rapporté à travers des études réalisées en 2007, des taux de Burn-out de 46,5\% et 30\%. De même Shanafelt et coll. ont démontré en 2012 que $65 \%$ des soignants américains avaient le Burn-out et que 40,2\% d'entre eux n'étaient plus satisfaits de leur travail (Shanafelt et al., 2012). En Australie, Arora et al. , (2013) ont retrouvé en 2013 un taux de $60 \%$ de Burn-out parmi les soignants des secteurs des urgences

En Afrique, Gharbi et coll. ont rapporté d'après une étude réalisée en Tunisie en 2012 que $54 \%$ des soignants des unités des urgences avaient le syndrome d'épuisement professionnel et $65 \%$ d'entre eux avait exprimé le souhait de changer de lieu de travail (Gharbi et al., 2012). Au Bénin, une étude réalisée à Cotonou en 2007 a montré que la prévalence du stress parmi les agents de santé était de 69,5\% (Hounkpè, 2007). A Parakou par contre aucune étude concernant ce sujet n'a été réalisée. L'objectif de ce travail est d'identifier les facteurs associés au Burn-out chez le personnel soignant des unités de soins intensifs de l'hôpital universitaire de Parakou.

\section{Milieu d'étude et méthodes}

Le Service d'Anesthésie-Réanimation et des Urgences (SARU), les unités de soins intensifs des Services de Pédiatrie et de GynécologieObstétrique et le bloc opératoire du Service de Chirurgie de l'hôpital universitaire de Parakou au Bénin ont servi de cadre d'étude. Il s'agit d'une étude transversale à visée descriptive et analytique avec recueil prospectif des données qui s'est déroulée du $1^{\mathrm{er}}$ mars au 30 juin 2015 et a reçu l'approbation du comité d'éthique institutionnel. La population d'étude était constituée des soignants (médecins, stagiaires internés, sages-femmes, infirmiers, aidessoignants) exerçant dans les unités de soins intensifs et ayant consenti à l'étude. La technique d'enquête quantitative a été celle utilisée pour la collecte des données. Cette collecte a été réalisée à l'aide d'un questionnaire préétabli administré à chaque enquêté pour une durée de cinq à huit minutes. Ce questionnaire a été complété par le Maslach Burn-out Inventory qui a servi à l'évaluation du syndrome d'épuisement professionnel des enquêtés. Les variables étudiées étaient : les données sociodémographiques, les facteurs de stress, la fréquence du burn-out et de ses composantes, les facteurs associés au burn-out, les mécanismes d'adaptation aux situations émotionnelles pénibles. Toutes les données collectées ont été traitées et analysées avec les logiciels Epi-Info version 7 et Epi Data version 3.1. Les variables quantitatives ont été 
exprimées en moyenne avec leurs écart-types. Les variables qualitatives exprimées en proportion avec leurs intervalles de confiance à $95 \%$ et en pourcentage. Les proportions ont été comparées avec le test de Chi-2 de PEARSON ou de FISHER selon le cas. La différence était considérée comme statistiquement significative pour une valeur de $\mathrm{p}<0,05$.

\section{Résultats}

\section{Données sociodémographiques}

Durant la période d'étude nous avions recensé 143 agents et parmi eux $123(86 \%)$ avaient consenti à l'enquête. L'âge moyen des soignants était de 34,4 \pm 9,3 ans avec des extrêmes de 21 et 58 ans. La tranche d'âge la plus représentée était celle des soignants de 21 et 30 ans $(46,3 \%)$ et une prédominance masculine $(57 \%)$ avait été notée avec une sex-ratio de 1,3 . Nous avons noté que 76 soignants (62\%) vivaient en couple et parmi eux $91 \%$ étaient monogames et 9\% étaient polygames, 46 soignants $(37 \%)$ étaient célibataires et 1 soignant $(1 \%)$ était divorcé. Plusieurs catégories professionnelles avaient été recensées : stagiaires internés (33\%), aidessoignants $(23 \%)$, infirmiers $(22 \%)$, médecins $(10 \%)$, infirmiers anesthésistes (7\%) et sages-femmes $(5 \%)$. Leur répartition dans les différents secteurs de soins a été précisée. Ainsi dans le Service de Gynécologie-Obstétrique nous avons dénombré 42 soignants $(34 \%)$ répartis en trois unités: soins intensifs $(20 ; 47,6 \%)$, bloc opératoire $(13 ; 31,0 \%)$ et salle d'accouchement $(9 ; 21,4 \%)$. Dans le Service d'Anesthésie-Réanimation et des Urgences il y avait 39 agents $(32 \%)$ répartis en trois unités : réanimation $(16 ; 41,0 \%)$, urgences $(14 ; 35,9 \%)$ et anesthésie $(9 ; 23,1 \%)$. Dans le Service de Pédiatrie nous avons recensé 31 soignants $(25 \%)$ et au bloc opératoire du Service de Chirurgie générale il y avait 11 agents (9\%). La durée d'ancienneté dans l'unité de soins a été également mentionnée. Elle était inférieure à un an pour $37 \%$ des soignants, comprise entre 1 et 5 ans pour $26 \%$ des soignants, comprise entre 5 et 10 ans pour $23 \%$ d'entre eux et supérieure à 10 ans pour $14 \%$ des enquêtés. Dans notre série 52 soignants (42\%) étaient satisfaits de leur situation socio-économique contre 71 soignants $(58 \%)$ qui ne l'étaient pas. Pour satisfaire les besoins de leur famille ces derniers exerçaient en plus de leur travail initial des activités secondaires notamment le commerce (19 ; $27 \%)$, les gardes dans des cliniques privées $(18 ; 25 \%)$, les soins à domicile $(9 ; 13 \%)$, les activités champêtres $(9 ; 13 \%)$, la vente de médicaments à domicile $(4 ; 6 \%)$, les cours à domicile $(2 ; 3 \%)$, le service informatique et bureautique $(2 ; 3 \%)$. Par contre 8 soignants non satisfaits $(11 \%)$ s'étaient contentés de leur situation et vivaient sans activité secondaire. Dans l'exercice de leur métier 37 soignants $(30,1 \%)$ ont développé une maladie. Il s'agissait de : l'ulcère gastrique $(21 ; 17,1 \%)$, l'hypertension artérielle $(10 ; 8,1 \%)$, 
l'asthme $(3 ; 2,4 \%)$, la cardiopathie $(1 ; 0,8 \%)$, la dermatose $(1 ; 0,8 \%)$, l'hémorroïde $(1 ; 0,8 \%)$.

\section{Facteurs de stress}

Dans notre série, le stress était lié à plusieurs facteurs. Le tableau I montre la répartition des soignants en fonction des facteurs de stress liés à l'organisation de travail.

Tableau I : Répartition des soignants en fonction des facteurs de stress liés à l'organisation de travail.

\begin{tabular}{cc}
\hline & Nombre/ Pourcentage \\
\hline Sentiment de pression au travail & $\mathbf{1 0 2}$ / 82,9 \\
Tâches multiples & $99 / 97,1$ \\
Surcharge de travail & $90 / 88,2$ \\
Charge énorme de travail & $70 / 68,6$ \\
Interruptions fréquentes des tâches & $45 / 44,1$ \\
Pression des supérieurs & $42 / 41,2$ \\
Annonce de décès & $\mathbf{9 0} / \mathbf{7 3 , 2}$ \\
Sonnerie des ambulances & $\mathbf{5 8}$ / 47,2 \\
Difficultés relationnelles au sein des équipes de & $\mathbf{4 4 ~ / ~ 3 5 , 8}$ \\
\hline
\end{tabular}

Le tableau II montre la répartition des soignants en fonction des facteurs de stress liés aux actes posés, au fonctionnent des équipements et aux secteurs d'activité.

Tableau II : Répartition des soignants en fonction des facteurs de stress liés aux actes posés, au fonctionnent des équipements et aux secteurs d'activité.

\begin{tabular}{|c|c|c|c|c|c|}
\hline & $\mathrm{N} / \%$ & & $\mathrm{~N} / \%$ & & $\mathrm{~N} / \%$ \\
\hline Actes posés & $56 / 45,5$ & $\begin{array}{l}\text { Fonctionnement } \\
\text { des Equipements }\end{array}$ & $39 / 37,1$ & $\begin{array}{l}\text { Secteurs } \\
\text { d'activité }\end{array}$ & $13 / 10,6$ \\
\hline $\begin{array}{l}\text { Massage } \\
\text { cardiaque }\end{array}$ & $24 / 19,5$ & $\begin{array}{l}\text { Fonctionnement du } \\
\text { respirateur }\end{array}$ & $14 / 11,4$ & Bloc opératoire & $7 / 5,7$ \\
\hline $\begin{array}{l}\text { Ventilation } \\
\text { mécanique }\end{array}$ & $10 / 8,1$ & $\begin{array}{l}\text { Manipulation de } \\
\text { l'aspirateur }\end{array}$ & $10 / 8,1$ & $\begin{array}{c}\text { Unité } \\
\text { d'anesthésie }\end{array}$ & $6 / 4,9$ \\
\hline $\begin{array}{l}\text { Intubation } \\
\text { trachéale }\end{array}$ & $9 / 7,3$ & $\begin{array}{c}\text { Indisponibilité de } \\
\text { l'Oxygène }\end{array}$ & $7 / 5,3$ & Aucun & $15 / 12,2$ \\
\hline $\begin{array}{l}\text { Drainage } \\
\text { pleural }\end{array}$ & $9 / 7,3$ & $\begin{array}{l}\text { Utilisation du } \\
\text { laryngoscope }\end{array}$ & $5 / 4,1$ & & \\
\hline $\begin{array}{l}\text { Drainage } \\
\text { abdominal }\end{array}$ & $4 / 3,3$ & $\begin{array}{l}\text { Manipulation de la } \\
\text { seringue électrique }\end{array}$ & $3 / 2,4$ & & \\
\hline
\end{tabular}

Les pathologies prises en charge dans les différents secteurs d'activité ont constituées également des facteurs de stress. Dans le Service d'AnesthésieRéanimation et des Urgences nous avons noté que : en anesthésie 100\% du personnel était stressé, en réanimation 11 soignants $(68,7 \%)$ étaient stressés et aux urgences 7 soignants (50\%) étaient stressés. Dans le Service de Gynécologie-Obstétrique nous avons objectivé que dans le secteur des soins 
intensifs 19 soignants (95\%) étaient stressés, au bloc opératoire 10 soignants $(76,9 \%)$ étaient stressés et en salle d'accouchement 6 soignants $(66,7 \%)$ étaient stressés. En pédiatrie, 18 soignants $(58 \%)$ étaient stressés et enfin au bloc opératoire du Service de chirurgie générale 9 soignants $(81,8 \%)$ étaient stressés.

\section{Fréquence du Burn out et de ses composantes}

Dans notre série 84 soignants $(68,3 \%)$ avaient le Burn out. Le tableau III donne la répartition des soignants en fonction de la fréquence du burn out et de ses composantes.

Tableau III: Répartition des soignants en fonction de la fréquence du Burn out et de ses composantes.

\begin{tabular}{cc}
\hline & Effectif / Pourcentage \\
\hline Burn out & $\mathbf{8 4}$ / 68,3 \\
Elevé & $31 / 25,2$ \\
Modéré & 53 / 43,1 \\
Composantes & $\mathbf{1 1 4}$ / 92,7 \\
Epuisement émotionnel & 59 / 48,0 \\
Elevé & 55 / 44,7 \\
Modéré & $\mathbf{9 4}$ / 76,4 \\
Dépersonnalisation & $56 / 45,5$ \\
Elevée & 38 / 30,9 \\
Modérée & $\mathbf{1 0 8}$ / 80,8 \\
Elevée & 80 / 65,0 \\
Modérée & 28 / 22,8 \\
\hline
\end{tabular}

\section{Facteurs associés au Burn-out}

Le tableau IV montre la répartition des soignants en fonction de la fréquence du Burn-out et le sexe, le type de famille, la qualification professionnelle et le secteur d'activités.

Tableau IV : Répartition des soignants en fonction de la fréquence du Burn-out et du sexe, du type de famille, de la qualification professionnelle et du secteur d'activités.

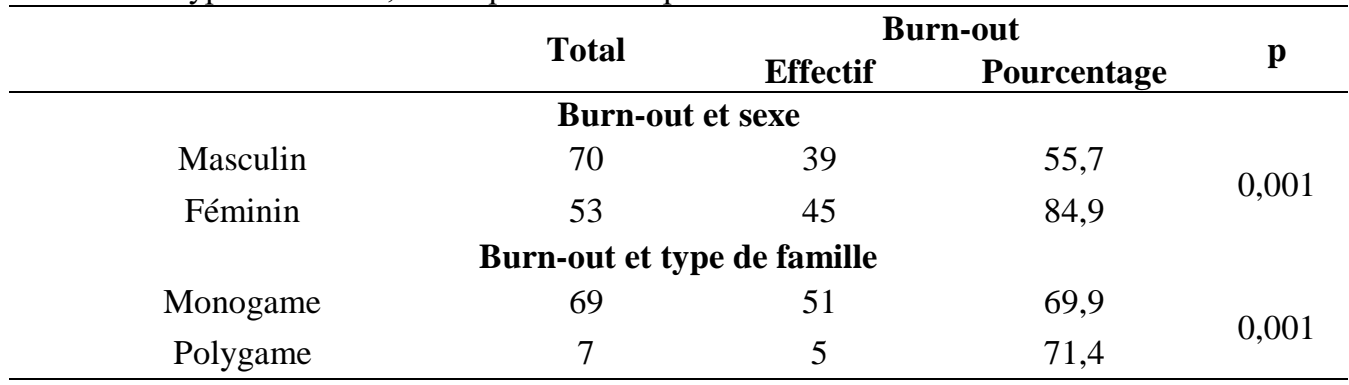




\begin{tabular}{ccccc}
\hline & Burn-out et qualification professionnelle & & \\
Stagiaires internés & 41 & 23 & 56,1 & \\
Aides-soignants & 28 & 23 & 82,1 & \\
Infirmiers & 27 & 21 & 77,8 & 0,001 \\
Médecins & 12 & 2 & 16,7 & \\
Infirmiers anesthésistes & 9 & 9 & 100 & \\
Sages-femmes & 6 & 6 & 100 & \\
& Burn-out et secteurs d'activités & & \\
Gynécologie-Obstétrique & $\mathbf{4 2}$ & $\mathbf{3 6}$ & $\mathbf{8 5 , 7}$ & \\
Soins intensifs & 20 & 17 & 85,0 \\
Bloc opératoire & 13 & 10 & 76,9 & \\
Salle d'accouchement & 9 & 9 & 100 & \\
SARU & 39 & 26 & 66,7 \\
Réanimation & 16 & 10 & 62,5 \\
Urgences & 14 & 7 & 50 \\
Anesthésie & 9 & 9 & 100 \\
Pédiatrie & 31 & 19 & 61,3 \\
Bloc opératoire chirurgie & 11 & 3 & 27,3 \\
\hline
\end{tabular}

Le tableau $\mathrm{V}$ montre la répartition des soignants en fonction de la fréquence du Burn-out et des conditions socio-économiques, des maladies apparues en cours d'exercice de métier de l'organisation du travail.

Tableau V : Répartition des soignants en fonction de la fréquence du Burn-out et des conditions socio-économiques, des maladies apparues en cours d'exercice de métier et de l'organisation du travail.

\begin{tabular}{|c|c|c|c|c|}
\hline & & \multicolumn{2}{|c|}{ Burn-out } & \multirow{2}{*}{ p } \\
\hline & & Effectif & Pourcentage & \\
\hline \multicolumn{5}{|c|}{ Burn-out et conditions socio-économiques } \\
\hline Non satisfaites & 71 & 55 & $\mathbf{7 7 , 7}$ & \multirow{2}{*}{0,01} \\
\hline Satisfaites & 52 & 29 & 55,8 & \\
\hline \multicolumn{5}{|c|}{ Burn-out et maladies apparues en cours d'exercice de métier } \\
\hline Maladies apparues & 37 & 29 & $\mathbf{7 8 , 4}$ & \multirow{8}{*}{0,02} \\
\hline Ulcère gastrique & 21 & 17 & 81,0 & \\
\hline Hypertension artérielle & 10 & 8 & 80,0 & \\
\hline Asthme & 3 & 1 & 33,3 & \\
\hline Cardiopathies & 1 & 1 & 100 & \\
\hline Dermatoses & 1 & 1 & 100 & \\
\hline Hémorroïdes & 1 & 1 & 100 & \\
\hline Aucune maladie & 86 & 55 & 64,0 & \\
\hline \multicolumn{5}{|c|}{ Burn-out et organisation du travail } \\
\hline Sentiment de pression au travail & 102 & 73 & 71,6 & \\
\hline Taches multiples & 99 & 70 & 70,7 & 0,000 \\
\hline Surcharge de travail & 90 & 70 & 77,8 & \\
\hline
\end{tabular}




$\begin{array}{cccc}\text { Charge énorme de travail } & 70 & 51 & 72,9 \\ \text { Interruption fréquente de tache } & 45 & 31 & 68,9 \\ \text { Pression des supérieurs } & 42 & 31 & 73,8 \\ \text { Annonce de décès } & 90 & 56 & 62,2 \\ \begin{array}{c}\text { Sonnerie des ambulances } \\ \text { Difficultés relationnelles au sein } \\ \text { des équipes de soins }\end{array} & 58 & 49 & 84,5 \\ \end{array}$

\section{Mécanismes d'adaptation aux situations émotionnelles pénibles}

Les mécanismes d'adaptation aux situations émotionnelles pénibles utilisés par les soignants étaient variés. Cent douze soignants $(91,1 \%)$ préfèrent en parler avec un proche, 62 soignants $(50,4 \%)$ font l'effort d'oublier ces situations pénibles vécues et préfèrent faire le vide autour d'eux, la consommation d'alcool est l'arme de dissuasion de certains d'entre eux (59; $48 \%)$, certains avaient manifesté le désir de changer de secteur d'activité (55; $44,7 \%)$, les loisirs sont préférés par certains $(34 ; 27,6 \%)$ et enfin un soignant préfère l'acharnement thérapeutique sur les prochains malades. Dans notre échantillon, 55 soignants $(44,7 \%)$ avaient manifesté le désir de changer de secteur et $19(15,4 \%)$ avaient envie de changer de métier. Les taux de burn out parmi ces soignants étaient respectivement de $76,4 \%$ et $89,5 \%$.

\section{Discussion}

\section{Facteurs de stress}

Les facteurs liés à l'organisation du travail étaient la principale cause de stress objectivés chez les soignants comme en témoignent les données de la littérature. Le facteur le plus évoqué était le sentiment de pression au travail. Ce sentiment donne lieu à des vécus différents qui semblent dépendre des sollicitations multiples dans l'accomplissement des tâches, la surcharge de travail, la charge énorme de travail, la pression des supérieurs et les interruptions fréquentes des tâches. Ces facteurs sont identiques à ceux rapportés par (Arora et al., 2013 ;Bertrand et al.).. La pression n'est pas forcement vécue de manière négative par tous les soignants. Certains soignants notamment ceux en début de carrière la perçoivent comme un vécu positif et source de motivation sur le plan professionnel et pour d'autres, elle apparaît comme source de stress et donc d'épuisement. Le même constat a été fait par (Scott et al. , 2008) où les infirmières en début de carrière ont estimé que les facteurs de stress sont cruciaux mais formateurs. Dans l'enquête de Derek et al., 2013, les soignants ont évoqué que la pression au travail est stressante. Ceci nous amène à demander si le travail en réanimation ne nécessite pas un choix et une vocation particulière permettant de s'adapter à une telle pression. L'étude de Laurent et al. ; (2007) réalisée sur le personnel soignant du SAMU met en avant un fort sentiment de vocation dans le service 
et la fatigue prend le sens d'un sacrifice pour mener à bien sa mission et plus celle-ci est exceptionnelle plus elle produira l'émulation de l'équipe.

L'autre réalité auquel la majorité de nos soignants était confronté est le manque de formation complémentaire notamment la spécialisation en médecine d'urgence. Dans les études menées par (Embriaco et al., 2007 ; Poncet et al., 2007 ; Gharbi et al., 2012) les unités d'accueil des urgences employaient des infirmières spécialisées contrairement à nos services où les soins sont administrés par des infirmiers non spécialisés en médecine d'urgence ou en réanimation. Il est donc probable que le stress soit induit par cette insuffisance de spécialisation. Il importe que ce vide soit comblé à leur niveau afin de les rendre plus aptes à prendre en charge les différentes détresses vitales.

L'effectif réduit en personnel, le manque de kits d'urgence et de consommables médicaux, les mésententes entre collègues, les incompréhensions avec les patients et leurs familles, l'incapacité des parents à honorer les médicaments, les difficultés liées à l'organisation de la gestion des urgences, la pression des encadreurs et le décès des patients apparaissaient comme sources de pénibilité sur le plan émotionnel. Ces mêmes facteurs ont été retrouvés par (Bertrand et al., 2007/2008).

Concerne les pathologies prises en charge dans les différentes unités de soins, certains soignants semblent être mieux aguerris pour y faire face que d'autres. Des pathologies ont été répertoriées par des groupes de soignants comme étant sources de stress et d'épuisement. C'est ainsi que les pathologies neurochirurgicales en l'occurrence celles des enfants étaient reconnues par les soignants du secteur d'anesthésie et du bloc opératoire de la chirurgie comme étant épuisantes. En réanimation, les urgences neurologiques étaient évoquées. Ceci s'expliquerait par le fait que la plupart des victimes reçus dans ces unités avaient un niveau socio-économique bas et les moyens mis en œuvre pour leur prise en charge étaient limités. Le pronostic de ces malades était souvent mis en jeu par défaut de médicaments et de soins appropriés. De plus, certains troubles neurologiques deviennent irréversibles et d'autres régressent progressivement laissant les soignants impuissants face aux souffrances des malades. Des troubles neuro-végétatifs peuvent se greffer au tableau clinique nécessitant de la part du personnel d'énormes efforts physiques et psychiques et constituent par conséquent des sources d'épuisement. Pour les grandes urgences neurochirurgicales comme les traumatismes vertébro-médullaires pour lesquels, le patient devrait bénéficier d'une intervention chirurgicale dans un très bref délai (dans moins de six heures au plus), le manque de moyens financiers, matériels fait souvent retarder l'intervention et déclenche au sein du personnel un épisode de stress en raison des conséquences qui en découleraient notamment la paralysie définitive. Dans le secteur des urgences du Service d'Anesthésie-Réanimation et des Urgences, outre les urgences 
neurologiques, les détresses respiratoires et les urgences cardio-vasculaires étaient identifiées comme stressantes. Dans les unités de soins intensifs du service de Gynécologie-Obstétrique, les soignants avaient évoqué les urgences neurologiques notamment les crises éclamptiques. Elles constituent une urgence obstétricale pouvant mettre en jeu le pronostic vital de la mère et de l'enfant d'où la mise en alerte de toute l'équipe des soins intensifs et du bloc opératoire toute situation qui constitue un facteur de stress. Au bloc opératoire du service de Gynécologie-Obstétrique, les urgences hémorragiques étaient plus évoquées car elles font courir toute l'équipe de garde et constituent par conséquent une source d'épuisement. Dans la salle d'accouchement, les dystocies, surtout lorsqu'elles nécessitent des manœuvres ou d'application d'instruments sont identifiées comme source de stress. En pédiatrie, les détresses respiratoires sévères, les urgences cardio-vasculaires et neurologiques, étaient identifiées comme facteurs de stress pouvant conduire au syndrome d'épuisement.

\section{Fréquence du Burn-out et de ses composantes}

La fréquence du Burn-out objectivée dans notre série était identique à celles de Shanafelt et al., (2012) ; Keller et al., (1989) en Amérique qui ont rapporté des fréquences respectives de $65 \%$ et $60 \%$. En, Tunisie, Gharbi et al., (2012) ont retrouvé, par contre une fréquence de 54\%. Le taux d'épuisement émotionnel retrouvé chez nos soignants était supérieur à ceux notés dans la littérature comme en témoignent les taux rapportés par (Lloyd et al., 46\% ;Bertrand et al., 2000 60\%; Arora et al.,. 60\% 2013). Le taux de dépersonnalisation retrouvé chez nos soignants était semblable à ceux rapportés par (Bertrand et al., 2000; Bell et al., 2002) qui étaient respectivement $78 \%$ et $66 \%$. Une fréquence plus élevée a été retrouvée par (Lloyd et al., 1994)93\%. Le taux de baisse d'accomplissement personnel retrouvé chez nos soignants était inférieur à celui obtenu par (Gillespie, 2003) $100 \%$ et supérieur à ceux obtenus par (Guinaud, 2006 (48\%) ; Bell et al., 2002. (34\%)).

\section{Facteurs associés au Burn-out}

Dans notre étude, le sexe, le type de famille, la qualification professionnelle, le secteur d'activité, les conditions socio-économiques, les maladies apparues dans l'exercice du métier et les facteurs liés à l'organisation de travail étaient liés au Burn-out. Ce constat a été fait par d'autres auteurs qui ont établi un lien entre les facteurs sociodémographiques et le Burn-out (Lloyd et al., 1994 ; Hui et al., 2012). Le personnel soignant féminin serait plus exposé du fait de ses occupations multiples. Contrairement aux hommes, elles seraient plus sensibles aux situations émotionnelles pénibles, ce qui justifierait leur forte représentation parmi les sujets atteints. Cette 
prédominance féminine a été retrouvée par des auteurs comme Shanafelt et al., (2012) ; Hui et al., (2012).

Vivre en couple, trouver quelqu'un avec qui partager ses peines sont des situations qui diminueraient les charges émotionnelles encaissées par les soignants. Les sujets en couple de notre échantillon étaient moins touchés que les célibataires. Mais ce n'était le cas des polygames qui étaient les premières victimes du Burn-out contrairement aux monogames. Cette vulnérabilité des polygames serait due aux charges conjugales qui leur pèsent dessus et quand, à ces dernières s'ajoutent les difficultés professionnelles, l'ensemble constitue finalement des facteurs de stress et par conséquent sources d'épuisement conduisant au Burn-out.

Les anesthésistes et les sages-femmes étaient les soignants les plus touchés. Le manque de personnel était un phénomène crucial qui pénalise le personnel anesthésiste. «Endormir un patient seul est très stressant 》 disaientils. C'est encore plus difficile pour les anesthésistes lorsqu'ils doivent prendre en charge des patients ASA 3 ou 4 ou lorsque des complications surviennent en cours d'intervention. S'agissant du cas des sages-femmes, les femmes hospitalisées dans les trois secteurs concernés par notre étude portent en général des pathologies graves et sont admises en situation d'urgence où il faut sauver deux vies (la mère et l'enfant) autant de facteurs qui augmente leur degré de stress.

Dans notre série, la plus faible fréquence du burn-out était notée pour les médecins. Ceci pourrait s'expliquerait d'une part, par leur niveau socioéconomique élevé et d'autre part par le fait qu'ils sont en général monogames et qu'ils ont une meilleure connaissance de la gestion des urgences. Ce constat est contraire à celui fait par Shanafelt et coll. qui ont rapporté que les médecins étaient plus exposés (65\%) (Shanafelt et al., 2012).

Le facteur organisationnel le plus prédictif du Burn-out dans notre étude était la sonnerie des ambulances. En effet, les soignants ne savant pas de quel type d'urgence il pourrait s'agir, certains propos reviennent souvent dans leurs langages à l'approche des ambulances : «eh encore...... ça pourrait être encore quel cas? Que Dieu fasse pour que ça ne soit pas une grande urgence! ». D’autres facteurs sont également évocateurs: les difficultés relationnelles au sein des équipes de soins, le sentiment de pression au travail notamment la fragmentation des tâches et les difficultés à annoncer les décès. Les mêmes facteurs ont été identifiés par plusieurs auteurs : Pour Bertrand et al., (2000); Arora et al., (2013); Marpinard ; (2007/2008) la surcharge quantitative et qualitative du travail, les difficultés relationnelles avec les confrères, les patients et leurs familles étaient les facteurs les plus retrouvés comme facteurs organisationnel et cause de stress. 


\section{Mécanismes d'adaptation aux situations émotionnelles pénibles}

Pour mieux gérer les effets du stress, la majorité des soignants parlaient de leur vécu. La plupart en font des confidences à leurs collègues et quelques peu en parlent aux psychologues. Pourtant au cours de notre enquête, la majorité des soignants affirmaient qu'il fallait un ' debriefing'" psychologique après chaque choc émotionnel vécu dans les services. Par contre, d'autres soignants préféraient s'investir dans le loisir : le cinéma, le sport, la lecture, la music, la prière, la promenade, ou s'efforcent d'obtenir la guérison des prochains malades par un acharnement thérapeutique afin d'oublier les échecs obtenus sur les autres malades.

Le loisir est un facteur protecteur contre le Burn-out d'après l'étude de Arora et al., (2013). Une autre étude faite par Marpinard ; 2007-2008 a retrouvé que l'absence de pratique sportive est un facteur favorisant le Burnout. Certains soignants, prenait l'alcool après les journées de travail afin d'oublier les vécus pénibles et de faire le vide. Ce constat a été fait par Goldberg et al., (1996) qui ont retrouvés que la fréquence du Burn-out était élevée parmi les soignants dont le niveau de consommation d'alcool était élevé.

Pour finir avec les situations stressantes, certains soignants avaient envie de changer de secteur, et d'autres voulaient changer de métier. Le même constat a été fait dans l'enquête européenne SeSMaT ayant couvert 2007 à 2008 (Marpinard ; 2007/2008) ; où 17\% des soignants ont exprimé leur intention d'abandonner leur métier. Et les causes les plus évoquées étaient les facteurs de stress.

\section{Conclusion}

La fonction soignante dans les services de soins intensifs du CHUD-B comporte une pénibilité physique et mentale ayant des effets délétères sur le rendement professionnel et la vie socio-familiale du personnel. Ce cadre de travail tressant induit chez eux un score élevé du burn out qu'il importe d'abaisser par la mise en œuvre d'un programme de prévention.

\section{References:}

1. Kirouac L. 2012. Du surmenage professionnel au burn-out : réponses sociales et issues individuelles aux difficultés du travail d'hier et d'aujourd'hui. Lien social et Politiques ; 1(67): 51-66.

2. Bureau International du Travail. 1993. Le stress dans le monde du travail: étude du Bureau International du Travail. Genève, BIT.

3. Embriaco N, Azoulay E, Barrau K, Kentish N, Pochard F, Loundou A, et al. 2007. High level of burn out in intensivists: prevalence and associate factors. Am J Respir Crit Care Med ;11(75):686-92. 
4. Poncet MC, Toullic P, Papazian L, Kentish-Barnes N, Timsit JF, Pochard F, et al. 2007.Burn out syndrome in critical nursing staff. Am J Respir Crit Care Med ; 11(75): 698-704.

5. Shanafelt TD, Boone S, Tan L, Dyrbye LN, Sotile W, Satele D et al. 2012. Burnout and satisfaction with work-life balance among us physicians relative to the general us population. Arch Intern Med ;172:1377-85.

6. Arora M, Asha S, Chinnappa J, Ashish D. 2013.Burnout in emergency medicine physicians. Emergency Medicine Australasia ; 98(125): 491-5.

7. Gharbi R, Bouchada N, Touil Y, Atig R, Fekih Hassen M, Elatrous S. 2012. Le burnout en milieu de réanimation et urgences : prévalence et facteurs de risque. Réanimation ; 22: 187-90.

8. Hounkpè SB. 2007. Etude du stress chez les agents de santé de deux centres hospitaliers au Bénin. Mémoire de certificat d'études de spécialités FSS: Université d'Abomey Calavi ;.

9. Bertrand F, Martinez P, Thiercelinl D, Fournier J.P, Van Elslande L, Romankiewiczl A, et al. 2000. Charge de travail des médecins des urgences: problème quantitatif ou qualitatif ? De la sérénité du médecin des urgences. Reanim Urgences scientifiques et medicales ; 19(9): 492-7.

10. Scott ES, Engelke MK, Swanson M.2008.New graduate nurse transition: Necessary or nice. (Applied Nursing Research; 21: 75-83.

11. Derek WJ, Martyn PD, Kathryn PD. 2013. Stress in Nurses: Stressrelated Affect and its determinants examined over the nursing day. ann behav med ; 45: 348-356.

12. Laurent A,Chahraoui K, Carli P. 2007. Les répercussions psychologiques des interventions médicales urgentes sur le personnel SAMU. Ann Med Psychol ; 165(1) : 570-8.

13. Marpinard M. 2007-2008. L'Enquête européenne SeSMaT : Mesure de l'épuisement professionnel chez les médecins et principes de prévention CHU de Toulouse. [Consulté le 19/08/2014]. Disponible sur http://www. dufmcepp.ups-tlse.fr

14. Keller KL, Koenig WJ. 1989. Management of stress and prevention of burn out in emergency physicians. Ann Emerg Med; 18: 42-7. (14)

15. Lloyd S, Streiner D, Shannon S. 1994. Burnout, depression, life and job satisfaction among Canadian emergency physicians. J Emerg Med; 12: 559-65.

16. Bell RB, Davison M, Sefcik D. 2002. A first survey: Measuring burnout in emergency medicine physician assistants.JAAPA; 15: 402, 45-8, 51-2. 
17. Gillespie M.2003.Burnout among nursing staff in accident and emergency and acute medicine: a comparative study. J Clin Nurs; 12: $842-51$.

18. Guinaud M. 2006. Evaluation du burn out chez les internes de médecine générale et étude des facteurs associés. Th: Med:Université Joseph Fourier (Grenoble).

19. Hui W, Wei Sun,Lie Wang. 2012.Factors associated with occupational stress among Chinese female emergency nurses. Emerg Med J; 29(8):554-8.

20. Goldberg R, Boss RW, Chan L. 1996. Burnout and its correlates in emergency physicians: four years' experience with a wellness booth. Acad Emerg Med; 3: 1156-64. 